
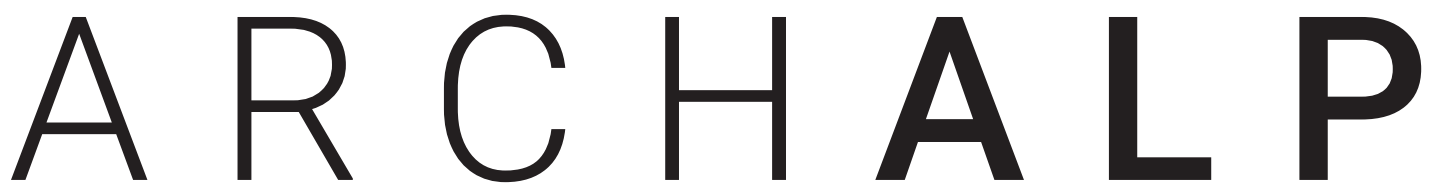

Rivista internazionale di architettura e paesaggio alpino / Revue internationale d'architecture et de paysage dans les Alpes / Internationale Zeitschrift für Alpine Architektur und Landschaft / Revija za alpsko arhitekturo in pokrajino / International journal of alpine architecture and landscape 
A Cervinia, tra la fine degli anni Quaranta e l'inizio degli anni Cinquanta del Novecento, prende corpo un dialogo pietrificato tra due edifici che segnano opposti immaginari dell'architettura alpina nella modernità: da un lato l'Albergo per ragazzi Pirovano disegnato da Franco Albini con Luigi Colombini, e dall'altro, a poche decine di metri, la Casa del Sole di Carlo Mollino.

L'Albergo Pirovano, di grande raffinatezza compositiva e costruttiva, come è noto muove dalla ripresa del tipo storico del rascard. La posizione concettuale di Albini è chiarissima: «l'edificio [...] si propone il problema dell'ambientamento nel paesaggio alpino, valendosi di quelle esperienze dell'architettura antica della Valle d'Aosta tuttora attuali e aderenti allo spirito moderno; [...] la programmatica limitazione ai mezzi costruttivi tradizionali e ai materiali tradizionali vuole accentuare l'esigenza di un profondo adeguamento alla natura e al costume del luogo. Non

In apertura

"Casa Capriata" alla Decima Triennale di Milano, 1953-1954.

Tutte le immagini provengono dal fondo Carlo Mollino, conservato presso gli Archivi della

Biblioteca Centrale di Architettura, "Roberto Gabetti" del Politecnico di Torino.

Fig. 1 Abitazione rurale a Champlas, Valle di Susa, anni Trenta, fotografia di Carlo Mollino. occorre certamente precisare che non si vuol parlare di architettura folcloristica, ma di un'architettura che non sia ambientalmente, e quindi urbanisticamente, indifferenziata e, ancora una volta, si vuol dire che l'architettura moderna non consiste nell'uso di materiali e di procedimenti costruttivi nuovi, ma che tutti i mezzi costruttivi sono validi in tutti i tempi purché logici e ancora efficienti» (Albini, 1951).

La Casa del Sole segue tutt'altre logiche, e il suo slancio dinamico verticale, il suo sbalzare e pendere in avanti in virtù degli aggetti crescenti dei balconi all'ascendere di quota sono stati vieppiù interpretati come una risoluta affermazione di una montagna quasi futurista e antinaturalistica, quella - per usare le parole dello stesso Mollino - dove l'architettura assurge al ruolo di «filtro [...] dove si entra ancora cittadini e si esce sciatori» (Red., 1948).

In realtà, se ci si sofferma a guardare il traliccio cementizio della facciata sud, avendo in mente i disegni di rilievo dell'architettura alpina storica che Mollino realizza negli anni Trenta, ci si accorge che c'è qualcosa di più sofisticato di un impulso vitalista verso una boccioniana città che sale in ambiente alpino. Il traliccio di facciata costituito dalle orizzontali dei terrazzi, sostenuti trasversalmente dalle travi accoppiate, le quali ammorsano i montanti verticali rastremati nei punti di giunto si configura infatti come una trasmutazione in cemento armato di una tipica configurazione alpina di matrice lignea.

Non è raro trovare nei progetti montani di Carlo Mollino questi dispositivi di manipolazione dell'architettura alpina storica all'opera. Un tema su cui in passato ha già lavorato Bruno Reichlin, ma che merita rinnovate indagini e attenzioni, alla luce dei nuovi valori e significati a cui oggi può assurgere il palinsesto materiale tradizionale. Come ha osservato Daniele Vitale, a partire dai progetti di Gino Levi Montalcini, nelle opere di Carlo Mollino e di altri torinesi dell'epoca «l'architettura sembra potersi liberare dell'alternativa tra mimesi e rivendicazione del nuovo, per scoprire una gamma più vasta e più complessa di possibilità. [...] la strada non sembra essere quella di riprendere gli elementi della tradizione per "attualizzarli", in base a un'esigenza "morale" e attraverso le sottigliezze di una rielaborazione poetica trattenuta e controllata [...]. Per i 
torinesi, gli elementi della tradizione paiono piuttosto essere "pretesti" di una esplorazione e di una manipolazione formale che cerca i suoi modi e le sue direzioni» (Vitale, 2003). Non quindi architetture e configurazioni costruttive portatrici di una «razionalità oggettiva», secondo l'accezione moderna di Giuseppe Pagano (Pagano, Daniel, 1936), ma materiali che, grazie all'interpretazione progettuale, determinando inediti campi di valore.

Qui di seguito il tentativo di enucleare alcuni dei dispositivi progettual-concettuali agenti nel cantiere montano di Carlo Mollino.

\section{Riprese e distorsioni}

Il processo progettuale che caratterizza molte delle opere montane di Mollino non segue quasi mai la logica lineare della ricerca di una soluzione a un problema, ma si caratterizza per una deviazione

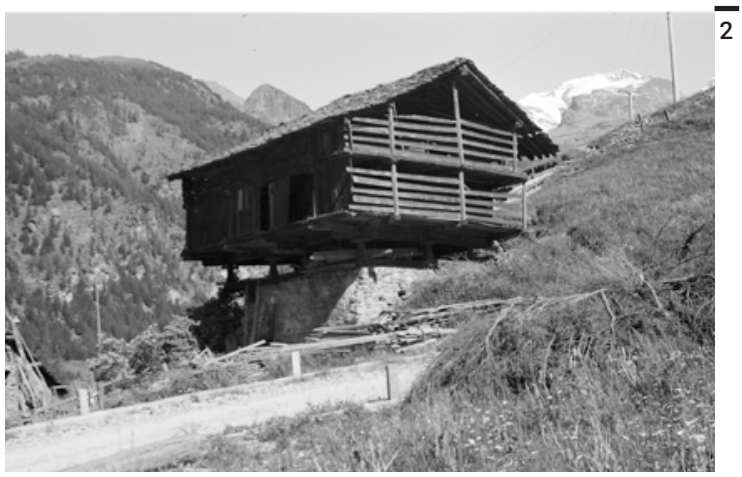

che crea un nuovo spazio valoriale, uno scarto che lo allontana dall'esito considerato come atto consequenziale. All'interno di questo approccio, egli dà vita ad un dispositivo di ripresa e distorsione di arche-

Fig. 2

Raccard Taleuc, Valle d'Ayas, Valle d'Aosta, anni Cinquanta, fotografia di Carlo Mollino.

Fig. 3 tavole di rilievo di architetture rurali valdostane, 1930.

$\overline{3}$

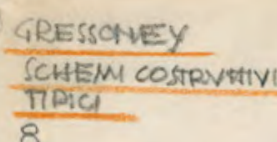

TIPO DI CASA

A TRVETYRA MISTA

P. TERRENO IN MMRATVRA I\& II IN LEQNO

FIG. 2
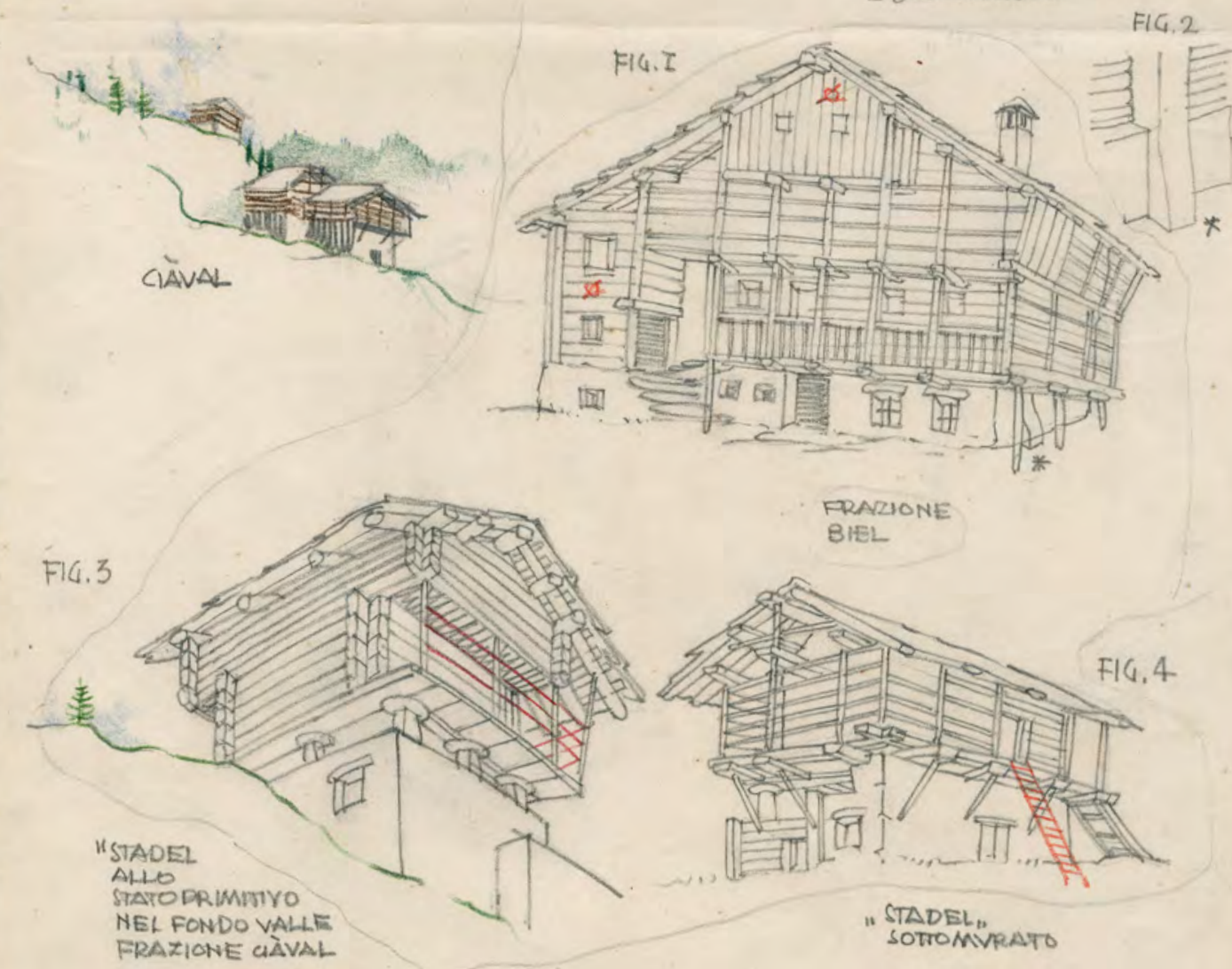

FRAZIONE

BIEL
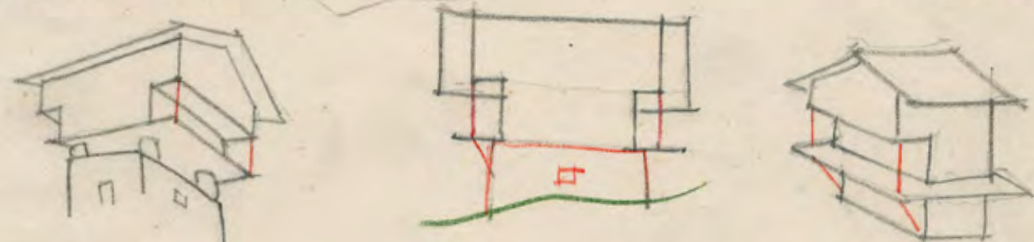
$\overline{4}$
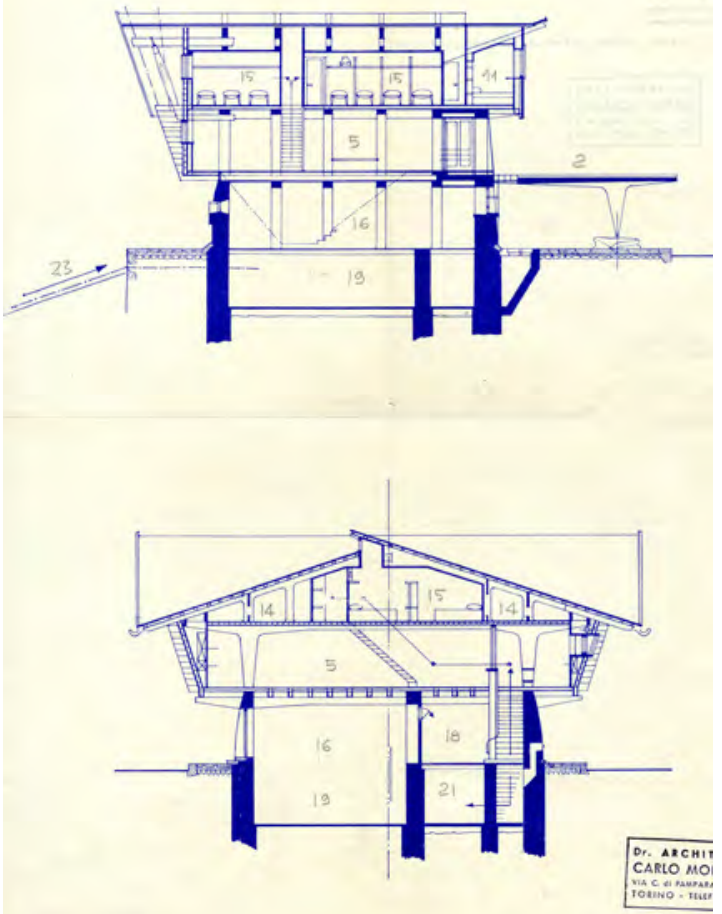

tipi e configurazioni convenzionali della tradizione storica, che diventano pretesto di reinterpretazioni generatrici di inediti significati tettonici e formali. Giò è particolarmente evidente nel processo ideativo per la Stazione della slittovia al Lago Nero a Sauze d'Oulx realizzata nel 1947, definita non a caso «uno degli edifici più tridimensionali dell'architettura italiana» (Kidder Smith, 1950), che muove proprio dalla ripresa del più archetipico edificio del contesto alpino per pervenire a una vera e propria «trasfigurazione della baita intesa non in senso folkloristico ma in sintesi con il senso dell'architettura d'oggi» (Red., 1948).

La plastica configurazione finale è l'esito di un lungo processo di modificazione e distorsione. Ai primi schizzi che delineano la tipologia della grangia tipica dell'alta Valle di Susa, seguono disegni in cui le geometrie della copertura e della terrazza progressivamente vengono deformate, e dove le facciate assumono caratteri e configurazioni compositive diverse tra loro. Il risultato è una «Vortäuschungsmaschine» - una macchina a finzioni, come ha scritto Reichlin (Reichlin, 1991) -, una costruzione à double face, che muta di aspetto a seconda del punto di osservazione. Verso valle l'edificio appare come un tradizionale e pesante Blockbau, ben radicato al suolo; verso monte il fronte è invece contrassegnato dalla straordinaria terrazza che si espande nello spazio e verso le piste - l'«approdo bianco», esito di un processo di progressiva deformazione dell'oggetto iniziale perfettamente riconoscibile nella sequenza degli schizzi-, nonché dallo sporto del tetto e dal volume superiore in legno che si protendono in avanti. La coesistenza di due immagini tanto diverse è assicurata dalle facciate laterali, che mediano il passaggio tra le due fronti, ma soprattutto dalla grande

Fig. 4

Slittovia al Lago

Nero, Sauze d'Oulx,

1946, sezioni.

Figg. 5-6

Slittovia al Lago Nero, Sauze d'Oulx, 1946 (foto Berkialla

Bressano).

5
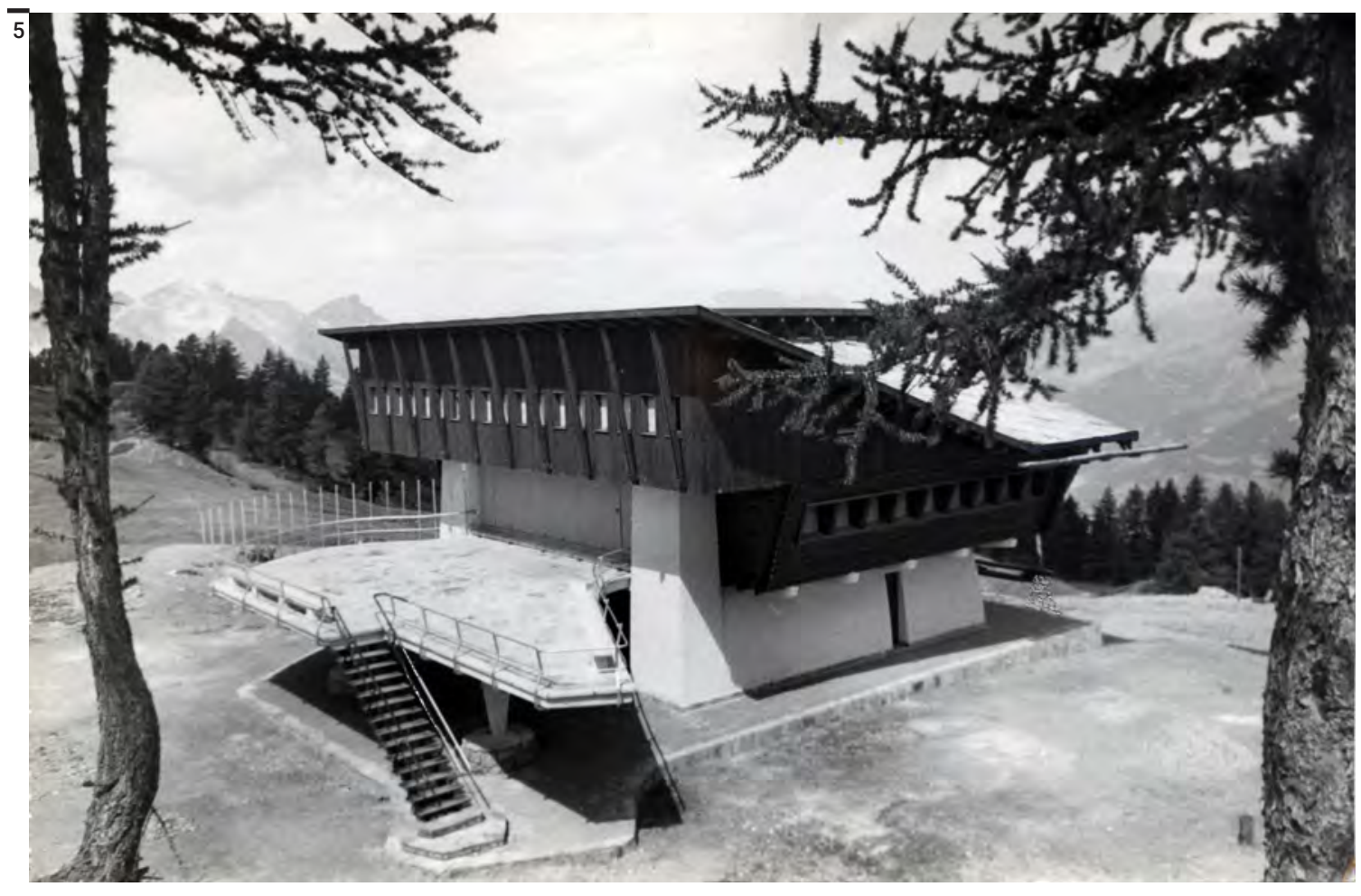
copertura a tre falde, che riunifica la composizione conferendo un aspetto tradizionale sul lato a valle - copertura a capanna - e modernista su quello a monte - monofalda che si apre verso il paesaggio. L'operazione di montaggio, quasi di collage, di configurazioni iconografiche così differenti, l'intenzionale ambiguità tra l'ossatura a scheletro in cemento armato e l'aspetto massivo dell'edificio all'esterno - con le travi in legno incastrate secondo la tecnica del Blockbau che fungono esclusivamente da tamponamento, come si può vedere nell'emblematico montaggio assonometrico, vero e proprio concept del progetto - mostrano una rottura rispetto ai codici elaborati dal moderno nei confronti della montagna, ma anche un'indifferenza verso il dibattito sulle preesistenze ambientali che coinvolge la cultura architettonica italiana durante gli anni Cinquanta.

\section{Smontaggio e rimontaggio, messa in cornice e objet trouvé}

Un'ulteriore forma di manipolazione delle figurazioni tradizionali a cui Mollino ricorre con frequenza è quella caratterizzata da un vero e proprio smontaggio e rimontaggio delle componenti architettoniche dei manufatti storici. Operazione che si basa sulla profonda conoscenza del patrimonio rurale che l'architetto torinese aveva coltivato attraverso i rilievi condotti sulle architetture alpine della Valle d'Aosta e dell'alta Valle di Susa.

Il rilievo non è mai però una statica rappresentazione della storia limitata alla sola dimensione co- noscitiva, quanto piuttosto una dinamica e intenzionale scomposizione-ricomposizione delle parti e delle componenti al fine di comprenderne le relazioni costruttive, le configurazioni statiche, le logiche tettoniche, al fine di riutilizzarle nel concepimento dei suoi dispositivi progettuali.

Emblematico da questo punto di vista è l'intervento per il raccard Garelli a Champoluc, assimilabile ad un'operazione artistica prossima a un ready-made, in cui la vecchia struttura, completamente decontestualizzata dalle funzioni e dalla localizzazione originaria, acquisisce nuovi significati, anche alla luce di alcuni elementi integrativi. L'antico raccard Taleuc, precedentemente localizzato in un sito a monte, viene completamente smontato, i pezzi vengono censiti e numerati e la struttura filologicamente ricostruita a valle, sopraelevata di un piano e posata su di un nuovo basamento in cemento armato e pietra a vista. Una lunga finestra a nastro viene inserita nello stacco - la chala$n e$ - tra il basamento e il raccard, e alla antica struttura viene accostata una nuova scala esterna di accesso in cemento armato e metallo simile a quella di un aeroplano. Un intervento di decontestualizzazione e di messa in cornice di un oggetto della tradizione, trattato appunto come un objet trouvé - che trova riscontri anche nello chalet incastonato sul Centro sportivo in verticale "Quota 2600" - che si configura quasi come un'operazione di arte concettuale ante litteram, dimostrando ancora una volta l'originalità della posizione di Carlo Mollino e la sua distanza dal dibattito architettonico italiano di quegli anni.

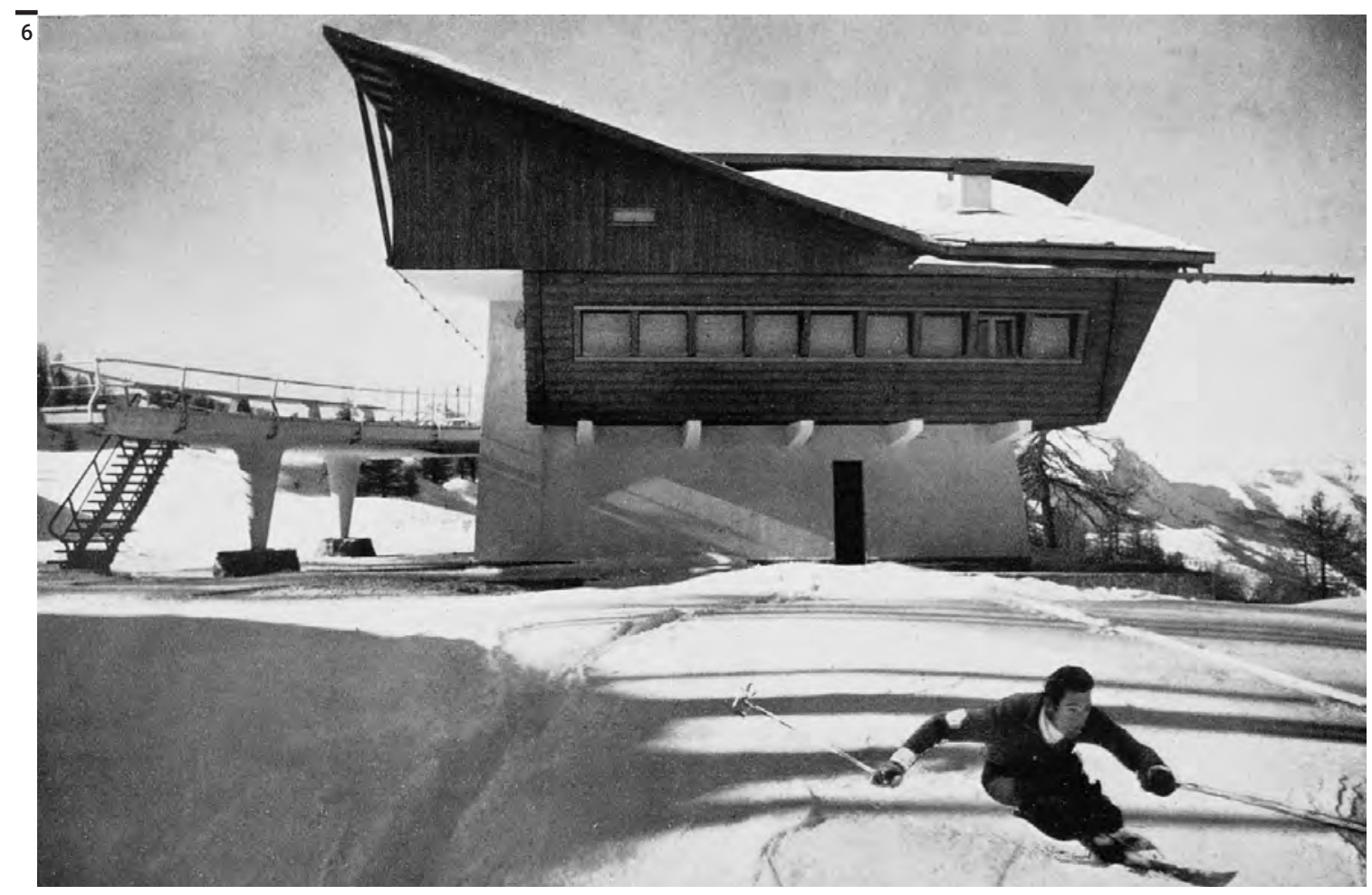




\section{Trasmutazioni metamorfico-metasemiche}

Nel progetto per il raccard Garelli troviamo un'ulteriore dispositivo che muove da una reinterpretazione degli elementi tradizionali. Si pensi alla soluzione dei nuovi pilastri a fungo che vengono riproposti come monoliti di granito rimodellati - non più ceppi di legno sormontati da una ruota in pietra, ma

Fig. 7

Progetto per la ricostruzione del raccard Taleuc per

Clotilde Garelli, Champoluc, 1963-1965.

Fig. 8

Progetto per la ricostruzione del raccard Taleuc per

Clotilde Garelli, Champoluc, 1963-

1965, dettaglio del "bolero".

Fig. 9 Casa Stefano Linot, Bardonecchia, 1951. quasi oggetti di design - perdendo il loro ruolo originario e la loro matrice funzionale (strutturale e di protezione dai roditori). Un processo dunque di "slittamento semantico" di una tipica soluzione in uso nel contesto montano attraverso la litificazione delle strutture originariamente lignee.

Lintervento di Mollino, a più riprese indagato e studiato, è stato anche spunto per un'approfondita riflessione di Bruno Reichlin sulla differenza tra interpretazione "stilistica" e interpretazione "funzionalista", mettendo a confronto i due differenti "funghi" utilizzati da Albini nel Pirovano di Cervinia e da Mollino nel progetto per il raccard Garelli a Champoluc: «Nel fungo di Albini la ruota in pietra è ridotta a lastra, ispessita al centro e disposta nella direzione delle travi - come se l'architetto delle presupposte funzioni d'origine, avesse ritenuta soltanto quella strutturale, consistente nella migliore ri- partizione sul trave di una carica puntuale. Non sarebbe difficile dimostrare come il rifugio Pirovano, nel suo insieme e sulla base delle esplicazioni fornite dall'architetto, rappresenti una rilettura moderna e in chiave "funzionalista" del rascard, conforme alle tesi enunciate dal Pagano nella celebre sezione sull'architettura rurale, alla Triennale di Milano del '36» (Reichlin, 1992). Mollino al contrario sostiene che «la permanenza di forme tipiche, rappresentative di un gusto, può originare cristallizzazioni su moduli fissi, veri standard di elementi strutturali, funzionali e formali. Questi elementi perdono la loro originaria ragione di esistenza, e rimangono come schemi astratti: stile» (Mollino, Valdacchino, 1947). Analogo dispositivo progettuale di litificazione avviene come si è visto nel traliccio della facciata della Casa del Sole a Cervinia, ma anche nella Casa Linot a Bardonecchia (1951) dove i doccioni e le grondaie lignee delle grange storiche vengono trasmutate in elementi di cemento armato.

\section{Sineddochi iperboliche}

Un ultimo dispositivo che Mollino riprende in modo quasi ossessivo in diversi progetti è quello dell'esasperazione di alcune figure costruttive, che da ele-

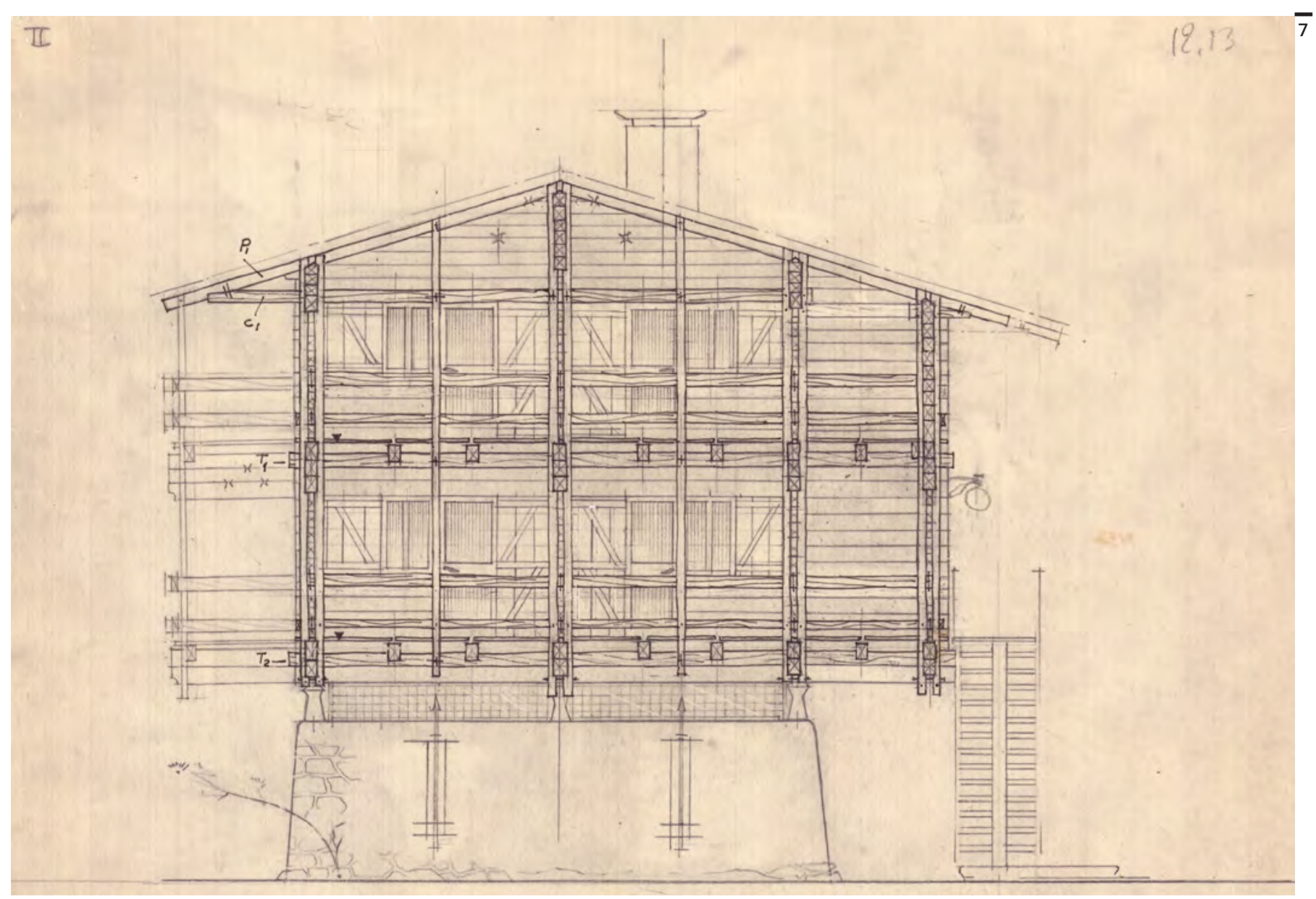




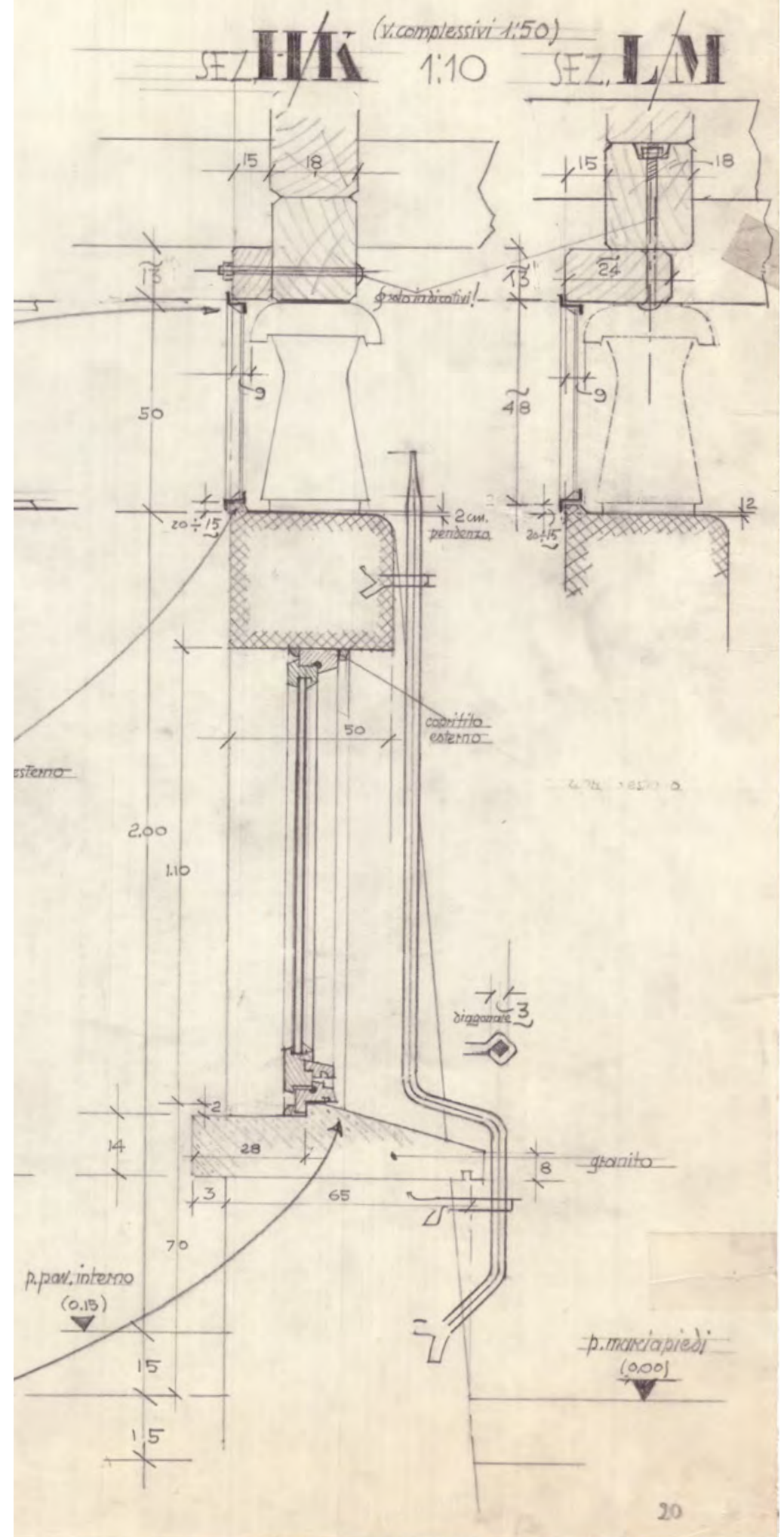

mento-parte di un tutto vengono amplificate e iperbolizzate fino a coincidere con la configurazione architettonica complessiva. Da questo punto di vista l'evoluzione del tema della casa triangolo basata sulla capriata rappresenta in modo emblematico come il dilatamento di un sistema costruttivo - il telaio strutturale ligneo della copertura - possa diventare il tema compositivo principale dell'architettura stessa. Se si osserva la sequenza di progetti e soluzioni che vanno dalla villa Dusio (1946) fino al prototipo elaborato per la X Triennale (1954), passando per la villa Carando (1947) e il progetto per il concorso Vetroflex-Domus (1951), si comprende la sua ricerca progettuale volta a far coincidere le caratteristiche statiche e costruttive con gli aspetti compositivi, distributivi e spaziali dell'intero manufatto.

Un altro "effetto" che Mollino sembra voler riprendere e rielaborare dal palinsesto dell'architettura storica montana è quello della sospensione da terra e del galleggiamento dei volumi, che - a partire dal sollevamento da terra dei raccard tradizionali, da lui a lungo studiati nei suoi sopralluoghi giovanili in Valtournenche e nelle valli Walser - pare superare la sola dimensione reinterpretativa della tradizione, evolvendo in un dispositivo totalizzante volto a produrre situazioni di vertigine e di straniamento tettonico. Aspetto che si riscontra in diversi progetti per architetture montane che si "staccano" dal suolo, come la casa di Lora Totino a Cervinia (1946), la scuola-colonia montana a Pré-Saint-Didier (1950), la casa del-

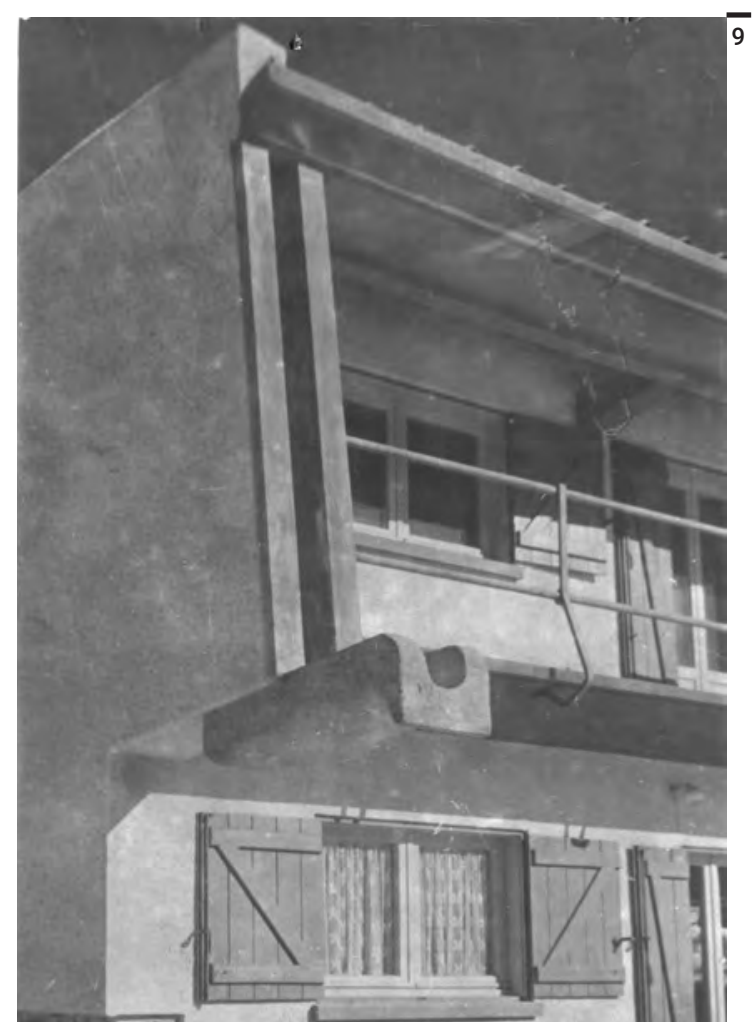




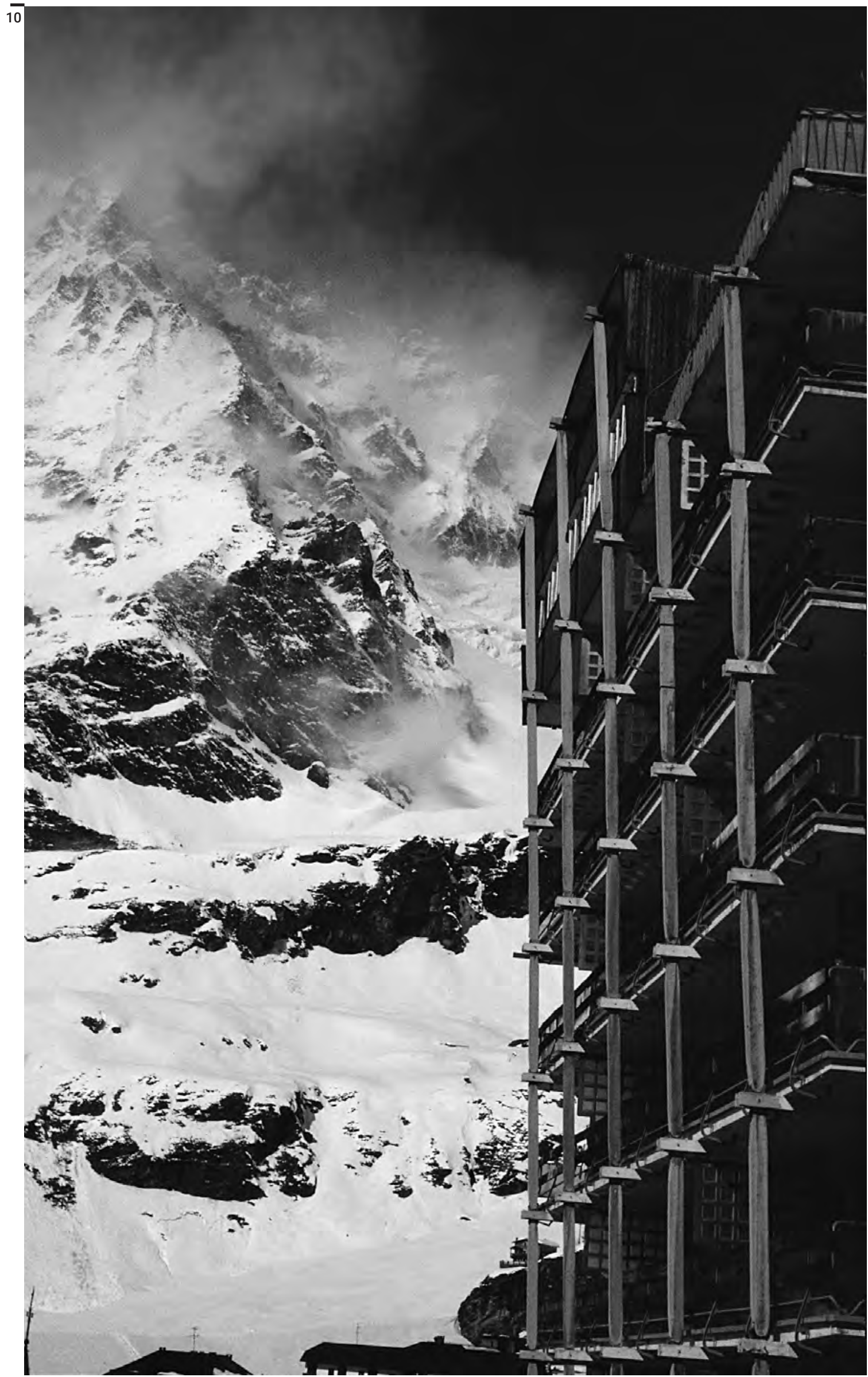


la FISI a Madonna di Campiglio (1951), fino all'opera più emblematica da questo punto di vista, la Casa Cattaneo, portata a compimento tra il 1952 e il 1954 sull'altopiano di Agra. Tali architetture sembrano concepite stravolgendo la configurazione bipartita tra basamento in pietra e volume superiore ligneo tipica delle costruzioni rurali. In genere, la parte basamentale viene metamorfizzata in elemento che sembra essere parte del suolo stesso, mentre il volume sovrastante sembra galleggiare grazie alla sospensione creata da uno sbalzo o dall'interposizione di elementi volti a svuotare il volume come terrazze, finestre a nastro, pareti vetrate. Ed è soprattutto in questi progetti che «scelta strutturale, sequenza spaziale, immagine e fruizione paesaggistica concorrono a un effetto di insieme rafforzandosi a vicenda» (Chiorino, Reichlin, 2006), quasi a simulare una sorta di montagnité intrinseca dell'architettura molliniana.

\section{Bibliografia}

Albini Franco (1951), «Albergo per ragazzi a Cervinia», in Edilizia Moderna, n. 47.

Bolzoni Luciano (1996), «Carlo Mollino e la montagna», in Ottagono, n. 11.

Brino Giovanni (2005), Carlo Mollino: architettura come autobiografia, Idea Books, Milano.

De Rossi Antonio (2016), La costruzione delle Alpi. Il Novecento e il modernismo alpino (1917-2017), Donzelli, Roma.

De Rossi Antonio, Moncalvo Enrico (a cura di) (2009), Cultura architettonica e ambiente alpino, Celid, Torino.

Kidder Smith George Everard (1950), L'Italia costruisce: sua architettura moderna e sua eredità indigenza, Edizioni di Comunità, Milano.

Milan Laura, Pace Sergio (2018), Carlo Mollino. L'arte di costruire in montagna. Casa Garelli, Champoluc, Mondadori Electa, Milano.

Mollino Carlo (1952), «Intervento alla Pro Cultura Femminile di Torino», in Atti e Rassegna Tecnica della Società degli Ingegneri e degli Architetti in Torino, n. 7.

Mollino Carlo (1954), "Tabù e tradizione nella costruzione montana», in Atti e rassegna tecnica della società degli ingegneri e degli architetti in Torino, n. 4.

Mollino Carlo, Vadacchino Franco (1947), Architettura, Arte e Tecnica, Chiantore, Torino.

Pace Sergio (a cura di) (2006), Carlo Mollino architetto, 1905-1973: costruire le modernità, Electa, Milano.

Red. (1948), «Stazione della slittovia al Lago Nero», in Domus, n. 226.

Red. (1948), «Una costruzione di oggi a Cervinia, che deve entusiasmare tutti gli sciatori. Centro sportivo in verticale "Quota 2600"», in Domus, n. 226.

Reichlin Bruno (1991), «Mollino in Bau und Schrift», in École d'Architecture de l'Université de Genève, Carlo Mollino. Baut in den Bergen, Catalogo della mostra, Basel.

Reichlin Bruno (1992), «Mollino sulle Alpi», in Casabella, n. 588.

Reichlin Bruno (1996), "Carlo Mollino nelle costruzioni e negli scritti», in Atti e rassegna tecnica della società degli ingegneri e degli architetti in Torino, n. 3, pp. 71-85.

Reichlin Bruno (1996), «Die Moderne baut in den Bergen», in Mayr Fingerle C. (a cura di), Neues Bauen in den Alpes, Architekturpreis 1995 - Architettura contemporanea alpina, Premio di Architettura 1995, Birkhäuser, Basel-Boston-Berlin. Vitale Daniele (1989), "Carlo Mollino», in Atti e Rassegna Tecnica della Società degli Ingegneri e degli Architetti in Torino, nuova serie, n. 11-12.

Vitale Daniele (2003), "Gino Levi-Montalcini e l'architettura torinese», in Levi-Montalcini Emanuele (a cura di), «Gino Levi-Montalcini. Architetture, disegni e scritti», in Atti e Rassegna Tecnica della Società degli Ingegneri e degli Architetti in Torino, n. 2.
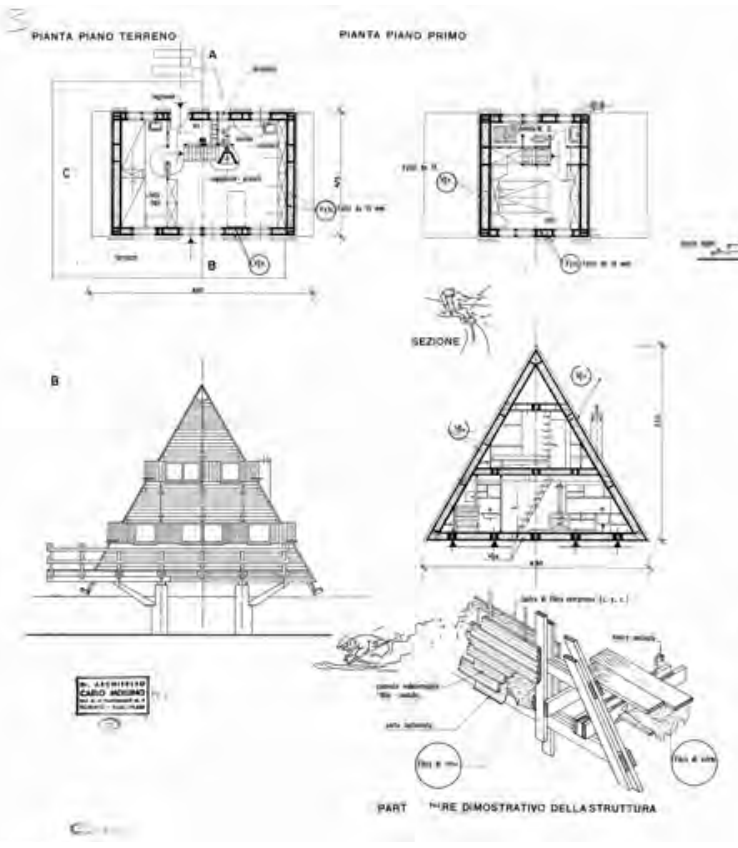
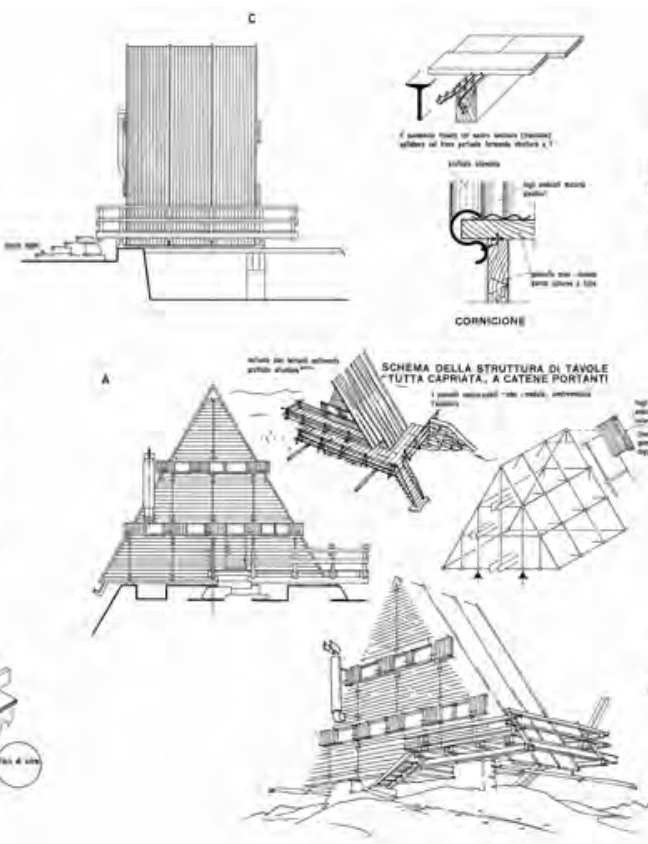
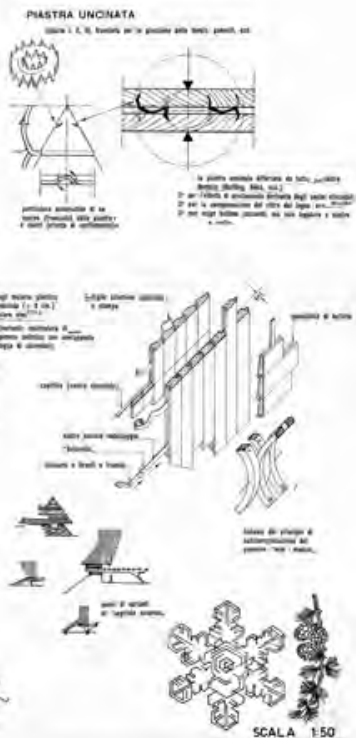